\title{
Association of Fatigue, Quality of Life and Functional Capacity in Breast Cancer Patients Receiving Adjuvant Chemotherapy
}

\author{
Shila B. Amarsheda ${ }^{1}$, Anjali R. Bhise ${ }^{2}$
}

${ }^{1} \mathrm{PhD}$ Research Scholar, Gujarat University, ESIC Model Hospital, Ahmedabad, Gujarat, India. ${ }^{2}$ Govt. Physiotherapy College \& Govt. Spine Institute, Gujarat University, Ahmedabad, Gujarat, India.

\begin{abstract}
Objectives: Cancer treatment related fatigue is a common and distressing symptom among breast cancer patients. With a greater longevity promote by advances in therapeutics \& early diagnosis, interest has shifted to the assessment of quality of life (QOL). The concept of functional capacity has been used extensively in disability and rehabilitation research as an indicator of an individual's ability to engage in physical activity. The aim of this study was to find the association of fatigue, QOL and functional capacity in post-operative breast cancer patients receiving adjuvant therapy. Materials and Methods: A total 30 participants who were receiving third cycle of chemotherapy were recruited from the Cancer Hospital. Facet fatigue questionnaire, FACT B questionnaire and 6-minute walk test (6MWT) were used for the assessment purpose of fatigue, QOL and functional capacity, respectively. Results: The Spearman's correlation confirmed that the fatigue is strongly associated with subscale score of Fact B and very strongly associated with total score of fact B, Fact G and TOI. There was moderate association between the fatigue and functional capacity. There were above average values in total and subscale scores of QOL but among all the subscale scores of QOL, breast cancer subscale score was having lower value compared to others and social well being score was higher. Conclusion: Fatigue is having strong association with quality of life and moderate effect with functional capacity in these patients. Specific breast cancer related health is having more association among other quality of life aspects and social well being is least affected.
\end{abstract}

Keywords: Breast cancer- Fatigue- Functional capacity- Quality of life

\section{Introduction}

Breast cancer is now the most common cancer in most cities in India, and $2^{\text {nd }}$ most common in the rural areas [1]. Cancer treatment related fatigue is a common and distressing symptom among cancer patients during and after treatment [2-3]. Patients describe the experience of cancer treatment related fatigue as being different from the fatigue experienced before cancer.

Cella and colleagues [4] have proposed a definition of fatigue that describe the symptom, common features and major characteristic. Fatigue is defined as an abnormal condition that persists for 2 or more weeks and occurs on most days. Common features may include reduced motivation \& interest in activities, exhaustion, apathy, generalized weakness, sleep abnormalities, irritability \& sadness.
According to Ream and Richardson [5], fatigue can be defined as a subjective, unpleasant symptom which incorporates total feelings ranging from tiredness to exhaustion creating an unrelenting overall condition which interferes with individual's ability to function to their normal capacity. To supplement this, NCCN panel has defined cancer-related fatigue as "An unusual, persistent, subjective sense of tiredness related to cancer or cancer treatment that interferes with usual functioning" [6]. Fatigue in patients with cancer is more severe, more distressing and less likely to be relieved by rest.

The etiology of fatigue is multi factorial and may include adverse effects of treatment, underlying disease, pain, sleep disorders, emotional distress, and a variety of metabolic and physiologic abnormalities. The ability to

\footnotetext{
Corresponding Author:

Dr. Shila B. Amarsheda

PhD Research Scholar, Gujarat University, ESIC Model Hospital, Ahmedabad, Gujarat, India

Email: shilaphysio@gmail.com
} 
maintain physical independence and to perform self-care activities can be compromised or lost if the integrity of physiological systems is not maintained. With a greater longevity promote by advances in therapeutics \& early diagnosis, interest has shifted to the assessment of quality of life. (QOL)

Major characteristics of fatigue include reduced energy that is disproportionate to activity level, distress often associated with reduced physical or intellectual performance that is casually related to the disease or its treatment and not related to a psychiatric disorder.

The concept of functional capacity has been used extensively in disability and rehabilitation research as an indicator of an individual's ability to engage in physical activity [7]. The physical ability to engage in activity is a universal self-care requisite [8] and is a component of health promotion, behaviors, particularly when undertaken with the aim of improving or maintaining one's health well-being [9-10].

Studies have shown that fatigue is most prevalent in breast cancer patients receiving adjuvant therapy but there are a smaller number of studies which shows that if there is an any association among fatigue, quality of life and functional capacity. In an effort to understand better the nature of cancer-related fatigue in patients receiving chemotherapy, an attempt has been made to find the association of fatigue, quality of life and functional capacity in post-operative breast cancer patients receiving adjuvant therapy.

\section{Materials and Methods}

\section{Study design and setting}

This observational study was carried out among the breast cancer patients who were receiving third cycle of chemotherapy from the cancer hospital, Ahmedabad between July 2019 to December 2019. Patients were recruited during third stage as the fatigue level declines over the three months interval [11].

\section{Sampling}

The pilot study was performed to calculate the sample size. Sample size of total 30 patients was calculated for the study purpose from the pilot study. The trial received ethical approval from Institutional ethical committee and written informed consent from all participants were taken. Eligible breast cancer patients met the following criteria: absence of metastasis or other malignancy, absence of chronic disease (such as hypertension. Kidney disease, heart problems, diabetes mellitus), poor psychological state, English or Gujarati or Hindi speaking or reading. Exclusion criteria were patients having past history of cancer, patients who were doing regular physical activities.

\section{Outcome Measures}

The FACIT scales are designed for patient selfadministration, but can also be administered by interview format. In this study, patients were interviewed at the third cycle of their chemotherapy treatment. For all FACIT scales and symptom indices, the higher the score the better the QOL.

\section{FACIT-fatigue Questionnaire}

FACIT-Fatigue questionnaire is a simple instrument for measurement of fatigue in cancer patients. The Functional Assessment of Chronic Illness Therapy - fatigue (FACITfatigue) scale is a 13-item instrument designed to assess fatigue/tiredness and its impact on daily activities and functioning in a number of chronic diseases. The instrument includes items such as tiredness, weakness, listlessness, lack of energy, and the impact of these feelings on daily functioning (e.g., sleeping, and social activities). This scale is reliable and valid measure of fatigue measurement in cancer patients [12].

\section{FACT-B Questionnaire}

The FACT-B is a breast cancer-specific HRQoL instrument of the FACIT system. The 37-item are divided into five subscales, namely physical (PWB), social/family (SWB), emotional (EWB), functional well-beings (FWB), and the additional concerns for breast cancer (BCS). Each item is rated on a 5-point Likert scale. Negatively worded items were recorded such that a higher score indicates a better HRQoL. The FACT-B total score is the sum of scores of all five subscales, the FACT-G score is the sum of PWB, SWB, EWB and FWB, while the Trial Outcome Index (TOI) is the sum of scores of the PWB, FWB and BCS. Missing values were imputed as the mean of observed items provided more than half of the items comprising a subscale were answered, i.e. the "half-rule". The FACT-B demonstrates ease of administration, brevity, reliability, validity, and sensitivity to change [13].

\section{6-Minute walk test (6MWT)}

Functional capacity was assessed with the 6-Minute walk test which assesses the submaximal level of functional capacity. The walking distance was 30 meters in length. In this test, the patient was asked to walk as far as possible in a period of 6 minutes. In cancer patients, the $6 \mathrm{MWT}$ seems to be as valid and reliable as in healthy elderly, cardiac and pulmonary patients [14].

\section{Results}

Data were analyzed using SPSS version 16 with 2-tailed probability tests with the level of significance set at $\mathrm{P}<.05$. Descriptive statistics (Table 1) were used for presenting the demographic data. In this study, total 30 participants were included in the study. Out of the 30 participants, $15(50 \%)$ patients were in the premenopausal stage and $15(50 \%)$ were in the post-menopausal stage. Of all the participants, $23(77 \%)$ patients were having breast conservation surgery either wide local excision (WLE) or lumpectomy and 7 (23\%) patients had mastectomy. The ongoing chemotherapy treatment consisted of drug regimens in the form of Doxorubicin and cyclophosphamide.

Spearman's rank-order correlation was used to explore association between the fatigue and quality of life total/ subscale score as well as between fatigue and 6-minute 
Table 1. Demographics

\begin{tabular}{lcc}
\hline & Mean & SD \\
\hline Age $($ Years $)$ & 46.3 & 8.74 \\
Height $(\mathrm{cm})$ & 156.77 & 5.58 \\
Weight $(\mathrm{kg})$ & 66.72 & 7.73 \\
BMI $\left(\mathrm{kg} / \mathrm{m}^{2}\right)$ & 27.31 & 4.33 \\
\hline
\end{tabular}

walk test. The strength of the correlations (r) was categorized as low $(0-.25)$, moderate $(>.25-.50)$, strong $(>.50-.75)$ and very strong $(>.75) .7$ Data are presented as means, standard deviations (Table 2), Spearman correlation coefficient (r) and 95\% confidence intervals (Table 3).

The Spearman's correlation confirmed that the fatigue is strongly associated with subscale score of Fact B and very strongly associated with total score of fact B, Fact $\mathrm{G}$ and TOI. There was moderate association between the fatigue and functional capacity measured by 6-minute walk test and there was no association between the 6 minutes walk test and quality of life total/subscale score. (Table 3) (Figure 1).

\section{Discussion}

This study explored the impact of fatigue on quality of life and functional capacity on breast cancer patients receiving adjuvant chemotherapy. In this study, an attempt has been made to focus the effect of fatigue on various aspects of quality of life in the form of physical wellbeing, social wellbeing, emotional wellbeing, functional wellbeing and specific breast cancer related health. As all the FACIT questionnaire shows the better quality of life with higher score, fatigue and Fact B questionnaire scoring with high value suggest less fatigue and good quality of life, respectively.

The result showed that there is strong correlation of fatigue with the subscale scores of quality-of-life measurement and very strong correlation with the total score. There was moderate correlation between fatigue and functional capacity measured by 6 -minute walk test. There were above average values in total and subscale scores of qualities of life but among all the subscale scores of quality of life, breast cancer subscale score was having lower value compared to others. Social well-being score was higher among all other subscale scores.

Cancer related fatigue (CRF) consists of different elements like physical, mental, emotional. Okuyama et al [15] have defined that complex structure of CRF and identified the physical, affective and cognitive issues in assessment of CRF.

The Quality of life for breast cancer patients commonly involves pain, recurrence fear and fatigue like other type of cancers. But, in breast cancer patients an altered sense of femininity, feeling of decreased attractiveness and arm swelling related problems concern additional problems. In this study, all the subscales have above average total score, however breast cancer subscale is comparatively low to other scores. It is suggesting that specifically in breast cancer patients overall quality of life will be affected because of the above mentioned factors.

Social well-being is having good score compared to other subscale scores in quality of life measurement. This is similar to other studies who also have founded that all functional scales were on a good level with the best function being social function. According to correlation study, social well-being is strongly associated with fatigue so if the patients' fatigue level will be high, it is going to affect the social well-being of the patients. The reason for the good social functioning can be good support from the family and friends. The patients may have loss the usual social interaction but they have received support by family and friends and therefore this loss was not perceived. This is similar to the study done by Olson et al [16] who have given possible explanation that the

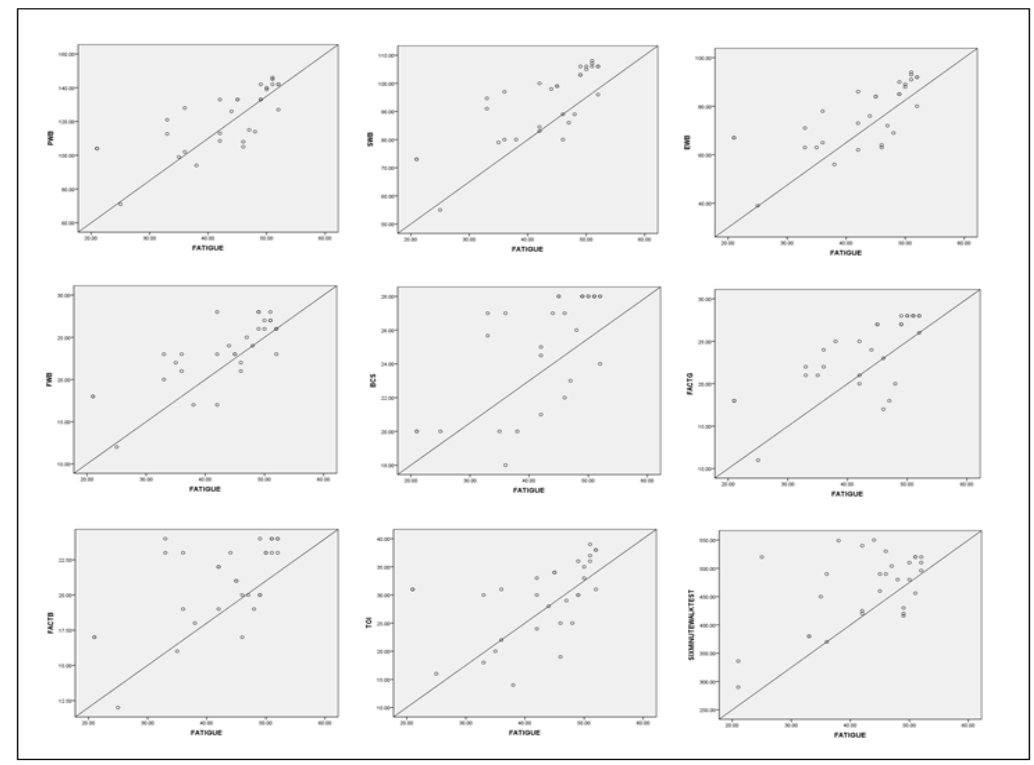

Figure 1. Scatter Diagram between Fatigue, Quality of Life (Total and Subscale Scores) and Functional Capacity 
Table 2. Descriptive Statistics for Facit Fatigue Questionnaire, Fact B Questionnaire and 6 Minute Walk Test

\begin{tabular}{lcc}
\hline & Mean (\%) & SD \\
\hline Fatigue & $42.70(82)$ & 9.07 \\
Physical well being (PWB) & $23.20(83)$ & 3.94 \\
Social well being (SWB) & $25.11(90)$ & 3.37 \\
Emotional well being (EWB) & $20.83(87)$ & 3.01 \\
Functional well being (FWB) & $23.60(84)$ & 4.39 \\
Breast cancer subscale (BCS) & $29.23(73)$ & 6.86 \\
Fact B & $121.84(82)$ & 18.49 \\
Fact G & $92.74(86)$ & 13.11 \\
Trial outcome index (TOI) & $76.03(79)$ & 13.48 \\
6 Minute Walk Test (meters) & 464.40 & 65.76 \\
\hline
\end{tabular}

patients receiving chemotherapy have already restricted their social interaction due to risk of infection and so that they did not associate social interaction due to fatigue. Bower [17] also supports that any kind of support may have beneficial effect on fatigue level of patient.

The support provided by the family also influences emotional well-being of the patients. In this study, fatigue was strongly correlated with emotional wellbeing. If the patients fatigue level will be high, emotional wellbeing will be negatively affected. Similar to this study, Yucel et. [18] al have found that fatigue negatively influenced the emotional functioning of the patients and subsequently their quality of life. The reason for the affection of emotional well-being of the patient can be the disease itself with that women are responsible for the maintenance of the home related activities, and worry about taking care of their family. Okuyama et al. [19] and Hofman et al. [20] found similar results, suggesting that the impact of fatigue on routine activities may extend in their emotional functioning.

In this study fatigue and functional capacity is moderately correlated. As the more fatigue score by Facit fatigue questionnaire suggest less fatigue level, it suggests that patients with more fatigue are having low functional capacity. In accordance with these, Curt et al. [21] in a survey of 379 cancer patients having a prior history of chemotherapy, low physical status was reported; they commonly described a significantly diminished energy level, a need to slow down from a normal pace, and a general sense of sluggishness or tiredness and perceived fatigue as the obstacle to live a "normal" life. Dimeo et al [22] have found that there is low correlation between physical performance and fatigue. The result can be because of the patients with fatigue may become accustomed to the fatigue and modified their expectation and their subjective experiences.

In cancer patients, most of the decline in functional capacity can be attributed to hypokinetic condition that happens because of prolonged physical inactivity. The decline in efficiency of multiple physiological system, initially cardio-respiratory and muscular systems are the cause of reduction in functional capacity. With continuation of inactivity, other physiological systems loss effectiveness and other impairments develop such as limited joint mobility, osteoporosis, impaired balance, paresthesia and lower pain threshold.

In cancer patients, common symptom is fatigue and universal in patients receiving adjuvant therapy. The proposed mechanism behind cancer related fatigue can be pro-inflammatory cytokines, hypothalamicpituitary-adrenal (HPA) axis dysregulation, circadian rhythm desynchronization, skeletal muscle wasting and genetic dysregulation. There are still little evidence supporting these proposed mechanisms [23].

Physiological fatigue can be divided into central and peripheral fatigue. Central fatigue is a factor with cancer, involves the central nervous system and the inhibition of neurological reflexes. Peripheral fatigue which is muscle specific can be divided into two components: muscle fatigue and muscle weakness. Oxidative stress as an underlying mechanism of the muscle weakness is developed because of cancer or chemotherapeutic agents. Nontargeted tissues, such as striated muscle, are severely affected by oxidative stress during chemotherapy, leading to toxicity and dysfunction [24].

Tabel 3. Spearman Correlation Coefficients between Fatigue, Quality of Life (Total and Subscale) and Functional Capacity

\begin{tabular}{|c|c|c|c|c|}
\hline & \multicolumn{2}{|l|}{ Fatigue } & \multicolumn{2}{|c|}{ Functional capacity (6 MWT) } \\
\hline & $\begin{array}{c}\text { Spearman Correlation coefficient } \\
\mathrm{r}\end{array}$ & $\mathrm{p}$ value & $\begin{array}{c}\text { Spearman Correlation coefficient } \\
\mathrm{r}\end{array}$ & $\mathrm{p}$ value \\
\hline FATIGUE & & & 0.3908@ & $0.0327 \#$ \\
\hline PWB & $0.7269 *$ & $<0.0001 \#$ & 0.0956 & 0.6151 \\
\hline SWB & $0.7146^{*}$ & $<0.0001 \#$ & 0.1152 & 0.5442 \\
\hline EWB & $0.5853^{*}$ & $0.0007 \#$ & 0.0835 & 0.6608 \\
\hline FWB & $0.7512 *$ & $<0.0001 \#$ & 0.1757 & 0.3529 \\
\hline $\mathrm{BCS}$ & $0.6233^{*}$ & $0.0002 \#$ & 0.0185 & 0.9225 \\
\hline Fact B & $0.7891 * *$ & $<0.0001 \#$ & 0.1321 & 0.4866 \\
\hline Fact $\mathrm{G}$ & $0.7932 * *$ & $<0.0001 \#$ & 0.1572 & 0.4068 \\
\hline TOI & $0.7672 * *$ & $<0.0001 \#$ & 0.1285 & 0.4985 \\
\hline
\end{tabular}

@moderate association; *strong association **very strong association \#statistically significant; (PWB, Physical well being; SWB, Social well being; EWB, Emotional well being; FWB, Functional well being; BCS, Breast cancer subscale; TOI, Trial outcome index) 
The result of the present study suggests that there is a strong association between fatigue and quality of life and moderate association between fatigue and functional capacity in breast cancer patients receiving adjuvant therapy. More intervention should be aim to reduce fatigue level in breast cancer patients which will help to improve various health aspects of quality of life and functional capacity. In future, research should be done at the different level of chemotherapy sessions and self-care activities can also be included to find the association with fatigue level and functional capacity.

In conclusion, there is an association of fatigue on various health aspects of quality of life in breast cancer patients receiving adjuvant chemotherapy. Fatigue is having strong effect on quality of life and moderate effect on functional capacity in these patients. Specific breast cancer related health is having more effect among other quality of life aspects and social well-being is least affected. In breast cancer patients, fatigue should be assessed systematically and appropriate measures should be taken to manage the patients' fatigue level. Proper rehabilitation should be done to provide optimum level of quality of life in these patients.

\section{Conflicts of Interest}

The authors declare no conflict of interest.

\section{Funding Statement}

This research did not receive any specific grant from funding agencies in the public, commercial, or not-forprofit sectors.

\section{Acknowledgements}

We would like to show our gratitude to Doctors and staff of the HCG Cancer Hospital, Ahmedabad for providing permission to carry out the study and giving support during this time.

\section{References}

1. Breast Cancer India. http://www.breastcancerindia.net.

2. Nail L, Jones L. Fatigue as a side effect of cancer treatment/; impact on quality of life. Quality of life-A Nursing challenge. $1995 ; 4: 8-13$

3. Schwartz A. Patterns of exercise and fatigue in physically active cancer survivors. Oncol Nurs Forum. 1998;25:458-91.

4. Cella D, Paterman A, Passik S, et al. Progress toward guidelines for the management of fatigue. Oncology. 1998;12:369-77.

5. Ream E, Richardson A. Fatigue: a concept analysis. International Journal of Nursing Studies. 1996 Oct;33(5):519529. https://doi.org/10.1016/0020-7489(96)00004-1

6. Atkinson A, Barsevick A, Cella D, et al. NCCN Practice guidelines for cancer-related fatigue. Oncol New York. 2000;14(11A suppl 10):151-61.

7. Vallbona C. Bodily responses to immobilization, Krusen's Handbook of Physical Medicine and Rehabilitation. Edited by FJ Kottke, GK Stillwell, JF Lehmann.

8. Orem D. Nursing: Concepts and practice, 2da. edic. NY: Mc Graw Hill. 1988.

9. Frank-Stromborg M. Health promotion behaviors in ambulatory cancer patients: facts or fiction?. InOncology nursing forum $2006 \mathrm{Jul} 1$ (Vol. 33, No. 4, p. C37). Oncology Nursing Society.

10. Walker SN, Sechrist KR, Pender NJ. The healthpromoting lifestyle profile: development and psychometric characteristics. Nursing research. 1987 Mar.

11. Vollrath M, Zenger M, Singer S, Einenkel J, Hinz A. The course of fatigue in patients with gynecologic and breast cancer. Journal of Gynecologic Oncology. 2013;24(3):280. https://doi.org/10.3802/jgo.2013.24.3.280

12. Yellen SB, Cella DF, Webster K, Blendowski C, Kaplan E. Measuring fatigue and other anemia-related symptoms with the Functional Assessment of Cancer Therapy (FACT) measurement system. Journal of Pain and Symptom Management. 1997 02;13(2):63-74. https://doi.org/10.1016/ s0885-3924(96)00274-6

13. Brady MJ, Cella DF, Mo F, Bonomi AE, Tulsky DS, Lloyd SR, Deasy S, Cobleigh M, Shiomoto G. Reliability and validity of the Functional Assessment of Cancer TherapyBreast quality-of-life instrument.. Journal of Clinical Oncology. 1997 03;15(3):974-986. https://doi.org/10.1200/ jco.1997.15.3.974

14. Schmidt K, Vogt L, Thiel C, Jäger E, Banzer W. Validity of the Six-Minute Walk Test in Cancer Patients. International Journal of Sports Medicine. 201302 26;34(07):631-636. https://doi.org/10.1055/s-0032-1323746

15. Okuyama T, Akechi T, Kugaya A, Okamura H, Shima Y, Maruguchi M, Hosaka T, Uchitomi Y. Development and Validation of the Cancer Fatigue Scale. Journal of Pain and Symptom Management. 2000 01;19(1):5-14. https://doi. org/10.1016/s0885-3924(99)00138-4

16. Olson K, Krawchuk A, Quddusi T. Fatigue in Individuals With Advanced Cancer in Active Treatment and Palliative Settings. Cancer Nursing. 2007 07;30(4):E1-E10. https:// doi.org/10.1097/01.ncc.0000281736.25609.74

17. Bower JE. Fatigue in cancer patients and survivors: mechanisms and treatment. Primary Psychiatry. 2005;12(5):53-7.

18. Yucel B, Akkaş EA, Okur Y, Eren AA, Eren MF, Karapınar H, Babacan NA, Kılıçkap S. The impact of radiotherapy on quality of life for cancer patients: a longitudinal study. Supportive Care in Cancer. 201404 12;22(9):2479-2487. https://doi.org/10.1007/s00520-014-2235-y

19. Okuyama T, Tanaka K, Akechi T, Kugaya A, Okamura H, Nishiwaki Y, Hosaka T, Uchitomi Y. Fatigue in Ambulatory Patients with Advanced Lung Cancer. Journal of Pain and Symptom Management. 2001 07;22(1):554-564. https://doi. org/10.1016/s0885-3924(01)00305-0

20. Hofman M, Ryan JL, Figueroa-Moseley CD, Jean-Pierre P, Morrow GR. Cancer-Related Fatigue: The Scale of the Problem. The Oncologist. 2007 05;12(S1):4-10. https://doi. org/10.1634/theoncologist.12-s1-4

21. Curt GA, Breitbart W, Cella D, Groopman JE, Horning SJ, Itri LM, Johnson DH, Miaskowski C, Scherr SL, Portenoy RK, Vogelzang NJ. Impact of Cancer-Related Fatigue on the Lives of Patients: New Findings From the Fatigue Coalition. The Oncologist. 2000 Oct;5(5):353-360. https:// doi.org/10.1634/theoncologist.5-5-353

22. Dimeo F, Stieglitz R, Novelli-Fischer U, Fetscher S, Mertelsmann R, Keul J. Correlation between physical performance and fatigue in cancer patients. Annals of Oncology. 1997 Dec;8(12):1251-1255. https://doi. org/10.1023/a:1008234310474

23. Mock V, Atkinson A, Barsevick A, Cella D, Cimprich B, Cleeland C, et al. NCCN Practice Guidelines for CancerRelated Fatigue. Oncology (Williston Park, NY). 2000 
Nov;14(11A):151-61.

24. Gilliam LA, St. Clair DK. Chemotherapy-Induced Weakness and Fatigue in Skeletal Muscle: The Role of Oxidative Stress. Antioxidants \& Redox Signaling. 2011 Nov;15(9):25432563. https://doi.org/10.1089/ars.2011.3965

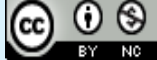

This work is licensed under a Creative Commons AttributionNon Commercial 4.0 International License. 